Im Verkehr mit von der Universität mit dem akademischen Grad Abgehenden, falle es als ein Fehler des Frziehungssystems häufig unangenehm auf, wie bedauerlich unschlüssig die größte Zahl dieser Leute sei über das Gebiet und die Stellung, für die sie geeignet wären. Es müsse deshalb während einer gewissen Zeit an den Universitäten die Aufmerksamkeit der Studenten auf die verschiedenen Gelegenheiten gelenkt werden, die das Gewerbsleben bietet, und den Studenten müßte ein klares Bild gegeben werden über die Aussichten und die Grenzen der Laufbahnen in der Technik sowie die Befähigungen, welche dafür nötig sind. Der Student müsse die Universität verlassen mit einem entschiedenen Zug zur Betätigung in einer bestimmten industriellen Richtung.

Es gäbe schließlich noch einen ganz bestimmten Wirkungskreis, in dem auch die kleineren Universitäten viel für die technische Entwicklung tun könnten, und zwar indem sie besondere Kurse in der Wissenschaft und der Technologie derjenigen Industrien einrichteten, die gerade in dem betreffenden Bezirk hoch entwickelt seien.

Die Universität in Ohio hätte sich z. B. jahrelang auf Gewinnung und Verarbeitung der Kohle spezialisiert, und die Universität in Maine richte zurzeit Kurse ein für die Papierfabrikation. Andere Universitäten und Kollegien seien für bestimmte Industrien sicherlich auch günstig gelegen. Wenn man diese Möglichkeiten auch nur in beschränktem Maße ausnutzen wolle, so hätten viele junge Leute, die sich für eine bestimmte industrielle Tätigkeit auszubilden gedächten, die Nittel hierzu in erreichbarer Nähe. Das Ergebnis würde die ständige Erhöhung des Zuflusses von jungen Leuten sein, die nicht blindlings in eine unbekannte Welt eintreten, sondern die mit offenen Augen und mit Willigkeit eine Tätigkeit auf sich nehmen, von der sie wüßten, daß sie ihr gewachsen seien.

\section{Die wichtigsten neuen Arzneimittel des Jahres 1915.}

\author{
Jahresbericht, im Auftrage der Fachgruppe für medizinisch- \\ pharmazeutische Chemie erstattet von Dr. F. Zernir. \\ (Eingeg. 21./1. 1916.)
}

Der Ausbruch des Weltkrieges hatte in der zweiten Hälfte des Jahres 1914 einen nicht zu verkennenden Einfluß auch auf die Produktion neuer Arzneimittel ausgeübt: es ist wohl kaum ein Präparat von Bedeutung in jenem Zeitraum herausgebracht worden. Im Jahre 1915 wurde das wieder anders. Der Markt der neuen Arzneimittel zeigt das gewohnte Bild; wenn die meisten größeren Firmen in der Auswahl der neu einzuführenden Mittel eine gewisse Vorsicht walten ließen, so entspricht das auch nur ibren Gepflogenheiten in den letzten Jahren vor dem Kriege. Bemerkenswert sind unter den neuen Präparaten einzelne, die gewissermaßen aus der Notwendigkeit des Krieges heraus geboren sind, wie z. B. die Ungeziefermittel. Daß unter dem Weizen auch diesmal manche Spreu sich findet, ist nicht auffällig. Insonderheit hat $\mathrm{B} \ddot{\mathrm{u}} \mathbf{r} \mathrm{g}$ i s viel mißverstandene und mißbrauchte Theorie über potenzierte Wirkung von Arzneigemischen ihren unheilvollen Anreiz auf manche Arzneimittelfabrikanten noch immer nicht eingebüßt, und so sehen wir, um nur ein Beispiel zu nennen, die Idee, ein Hypnoticum mit einem Antineuralgicum und einem Narcoticum zu kombinieren, in mancherlei Abarten nach wie vor abgewandelt. Es kann natürlich nicht die Aufgabe dieser Zeilen sein, alle derartigen Präparate und noch viel weniger alle die neu eingeführten Arzneispezialitäten hier aufzuzählen, die weder chemisch, noch therapeutisch etwas Neues bieten. Der vorliegende Bericht soll sich vielmehr darauf beschränken, die wichtigsten und charakteristischsten Vertreter unter den Neuerscheinungen auf dem Arzneimittelmarkt kurz zu besprechen, um so ein möglichst übersichtliches und scharfes Bild von den Fortschritten auf diesem Gebiete zu geben.
Unter den zur äußeren Antisepsis bestimmten Präparates sei zuerst das $M$ o $r$ o $n$ a l (Heyden) genannt. Moronal ist basisch formaldehydschwefligsaures Aluminium; es wird als fester Ersatz der gelösten essigsauren Tonerde empfohlen, der unbegrenzt haltbare und durch Kochen sterilisierbare Lösungen liefert, welche die Haut nicht macerieren. Klinische Beobachtungen wurden bisher nicht veröffentlicht. Ein ähnliches Präparat brachte das Vorjahr im A c e t o f or m (Kalle), essig-citronensauren Aluminiumhexamethylentetramin.

Aus den Angaben über die Zusammensetzung des V e r $\mathrm{n}$ i s a n m p u r u m (Dr. W. Nießen, Köln) ist nur ersichtlich, daß es eine ,Verbindung von Jod, Phenol und Campher". darstellen soll, vermutlich also wohl ein Gemisch; es soll in der Wundbehandlung unverdünnt oder verdünnt angewendet werden; die dem Vernisan nachgerühmte Tiefenwirkung würde sich aus der Zusammensetzung leicht erklären ${ }^{1}$ ).

Eingehende Versuche von $\mathrm{R}$ o st haben ergeben, da $\boldsymbol{B}$ aus gewissen Mineralölen sich Substanzen von ausgesprochen bindegewebsanregender Kraft isolieren lassen, die ihrer chemischen Natur nach als ungesättigte, partiell hydrierte Kohlenwasserstoffe von der Art etwa der Terpene und Polyterpene anzusprechen sind. Diese sind in den verschiedenen Mineralölen des Handels je nach dem Grade von deren Reinigung entweder in $\mathrm{zu}$ geringer Menge vorhanden, oder es finden sich neben ihnen noch andere störende Beimengungen. Diese Beobachtungen leiteten bei der Einführung des Granugenols (Knoll), eines Mineralöls, das gerade so weit gereinigt wurde, daß die schädlichen Bestand teile entfernt wurden, während ein hinlänglicher Anteil an jenen biologisch wirksamen Stoffen erhalten blieb. Bemerkenswert ist die Tatsache, daß die isolierten Träger der biologischen Wirkung für sich oder in indifferenter Lösung jene Wirkung auf das Gewebewachstum nicht in dem Maße besaßen, wie wenn man sie in Verbindung mit noch einem Tèil ihrer ursprünglichen Begleitstoffe belassen hatte. Granugenol soll in der Wundbehandlung zur Reinigung der Wunden und Beschleunigung ihres Schlusses Anwendung finden ${ }^{2}$ ).

Auf dem gleichen Prinzip wie Granugenol dürfte wohl auch das B e n e g r a n (Pharm. u. chem. Spezialgesellschaft m. b. H., Berlin) beruhen; es ist eine wachsartige Substanz, die im wesentlichen eine Lösung von Kautschukharz in niedrigschmelzenden Kohlenwasserstoffen (Paraffin und Vaseline) darstellen soll und in erhitztem Zustand auf die Wunden aufgetragen wird. Auch hier resultiert eine Beschleunigung der Wundheilung ${ }^{3}$ ).

Ein nach Angaben von Frerich s nach bisher nicht bekanntgegebenem Verfahren hergestelltes $\mathrm{W}$ i s $\mathrm{m}$ u $\mathrm{t}$ $\mathrm{subacetat}$ (Merck), $\mathrm{CH}_{3} \mathrm{COO} \cdot \mathrm{BiO}$, mit rund $82 \%$ $\mathrm{Bi}_{2} \mathrm{O}_{3}$, soll als Wundstreupulver in geeigneter Verdünnung, auch als Zusatz zu Wundsalben Anwendung finden ${ }^{4}$ ).

Im Gegensatz zu dem eben genannten Mittel, über das klinische Veröffentlichungen bisher fehlen, hat das Wundstreupulver Leuk oz on (Calcium perboricum ,Byk“" c. Talco $50 \%$ ) (Chem. Werke vorm. Dr. H. Byk, Oranienburg) im verflossenen Jahr in der medizinischen Literatur mehrfach empfehlende Erwähnung gefunden. Die Vorzüge dieses nach D. R. P. 248683 bzw. 286517 dargestellten Präparates beruhen darin, daß es langsam und kontinuierlich aktiven Sauerstoff abspaltet; hervorgehoben wird namentlich auch die stark desodorierende und austrocknende Wirkung des Leukozons sowie seine Eigenschaft, die Wundheilung erheblich abzukürzen ${ }^{5}$ ).

1) D. Med. Wochenschr. 41, 487; 860 [1915]

2) Deutsche Zeitschr. f. Chirurgie 133, 344 ff. [1915]; Münchn. Med. Wochenschr. 62, 859, 860 [1915]; Württ. med. Korr.-Blatt 1915, Nr. 21; Med. Klinik 11, 1031 [1915]; D. Med. Wochenschr. 41, 1162 [1915].

3) Berl. Klin. Wochenschr. 52, 941 [1915].

4) Apotheker-Ztg. 30, 156, 182 [1915]; Angew. Chem. 28, II, 274 [1915].

5) D. Med. Wochenschr. 41, 461 [1915]; Med. Klinik 11, 645 [1915]; Berl. Klin. Wochenschr. 52, 665 [1915]; Münchn. Med. Wochenschr. 62, 1236 [1915]. 
Utber den Wert einer Trinkwassersterilisation im Felde durch chemische Mittel sind die Ansichten noch geteilt; vielfach wird dem einfachen Abkochen des Wassers der Vorzug gegeben. Will man aber eine Sterilisation durch Chemikalien gelten lassen, so ist wohl das D e s a z on (Bayer) als das nach den derzeitigen Erfahrungen hierfür geeignetste Mittel anzusehen.

Es ist bekannt, daß sich mit Chlorkalk eine vorzügliche Trinkwassersterilisation erzielen läßt; als störend wurde dabei nur der Geruch und Geschmack des überschüssigen Chlorkalks empfunden. Nach D. R. P. $260653^{5 \mathrm{a}}$ ) ist es gelungen, dem abzuhelfen. Zur Verwendung gelangt ein $75 \%$ iger Chlorkalk, von dem schon $0,2 \mathrm{~g}$ genügen, um in 11 Wasser alle pathogenen Bakterien abzutöten. Der überschüssige Chlorkalk wird dann durch Zufügung von $0,35 \mathrm{~g}$ Ortizon (Wasserstoffsuperoxyd-Harnstoff) zerstört im Sinne der Gleichung

$$
\mathrm{CaOCl}_{2}+\mathrm{H}_{2} \mathrm{O}_{2}=\mathrm{CaCl}_{2}+\mathrm{H}_{2} \mathrm{O}+\mathrm{O}_{2} \text {. }
$$

Unter dem Namen D e s a z o n kommen besondere Feldpackungen in den Handel, die je 10 Glasröhrchen mit $0,2 \mathrm{~g}$ Chlorkalk bzw. 0,35 g Ortizon und eine entsprechende Gebrauchsanweisung enthalten ${ }^{6}$ ).

Daß im übrigen mit Mitteln zur chemischen Trinkwassersterilisation nach wie vor ein ziemlicher Unfug getrieben wird, sei beiläufig erwähnt; im verflossenen Jahre wurde namentlich ein unter dem Namen $K$ a $t$ a $c$ id angepriesenes derartiges Präparat aus Wasserstoffsuperoxyd-Harnstoff und Citronensäure als völlig unwirksam gebrandmarkt ${ }^{7}$ ).

Ähnlich wie bei der Trinkwassersterilisation gilt auch für die Zwecke der Ungeziefer-, besonders Läusevertilgung im Felde die Hitze als das beste und billigste Vernichtungsmittel und die Heeresverwaltung hat in ihren zahlreichen Entlausungsanstalten die entsprechenden Folgerungen gezogen. Immerhin bietet sich für wirklich brauchbare chemische Präparate hier noch ein weites Feld. Von den vielen, mit mehr oder weniger Recht als wirksam empfohlenen Mitteln verdienen Erwähnung das G 1 o b o l (Fritz Schulz jr., Leipzig) p-Dichlorbenzol, im Handel in Stoffbeuteln, und namentlich $\mathrm{L}$ a u s of a $\mathrm{n}$ (Bayer), Cyclohexanon mit etwas Cyclohexanol (vgl. D. R. P. Anm. B 68720; Angew. Chem. 27, II, 405 [1914]), im Handel als 20\% Pulver und 20\% Lösung. Lausofan wirkt stark abtötend auf Ungeziefer aller Art (Läuse, Flöhe, Wanzen) sowohl durch direkte Berührung wie durch Dunstwirkung. Die Anwendung ist bequem, der Geruch wird nicht als unangenehm empfunden; schädliche Nebenwirkungen wurden nicht beobachtet ${ }^{8}$ ).

Bei einem anderen Ungeziefervertilgungsmittel $\mathrm{Pla}-$ g i n, das, wie die Analyse ergab, im wesentlichen aus kieselfluorwasserstoffsaurem Natrium bestand, waren dagegen die Reizwirkungen so erheblich, daß sein weiterer Vertrieb behördlich verboten wurde ${ }^{9}$ ).

Es sei an dieser Stelle auch des $P$ u $I$ is $f I$ a e n s hydrargyri (W. Mielck, Hamburg) gedacht. Nach Un $n$ a ist das für die Wirkung der grauen Quecksilbersalbe Wesentliche die teilweise Oxydation des Quecksilbers zu Oxydul bzw. Oxyd und zu Oleaten; diese Verbindungen übertragen dann immer weiter Sauerstoff auf neues Quecksilbermaterial. Danach ist also für die percutane Anwendung von metallischem Quecksilber die Herbeischaffung von lose gebundenem aktiven Sauerstoff von Wichtigkeit oder von viel Luft, deren Sauerstoff dann aktiviert werden kann. Unna erreicht beides, indem er Lykopodium, das Prototyp der durch ihren hohen Luftgehalt ausgezeichneten ,Gleitpuder", mit Terpentinöl, dein vorzüglichen Katalysator des Luftsauerstoffs, imprägniert und das erhaltene Pulver mit $33{ }_{3} \%$ metallischem Quecksilber verreibt. Dieser Pulvis fluens hydrargyri ist leicht auf der Haut verreibbar, kaum

5 a) Angew. Chem. 26, II, 462 [1913].

6) Hygien. Rundschau 273 [1915]; D. Med. Wochenschr. 41, 127 [1915]; Pharm. Zentralh. 1915, Nr. 20.

7) D. Med. Wochenschr. 41, 536 [1915]; 42, 98 [1916].

${ }^{8}$ D. Med. Wochenschr. 41, 861, 1464[1915]; Med. Klinik 11, 645 [1915].

9) Berl. Klin. Wochenschr. 52, 552 [1915]; Apotheker.Ztg. 30, 295 u. 390 [1915]; Angew. Chem. 28, II, 431 [1915]. sichtbar und nicht fettend; außer zu Quecksilberkuren leistet er auch als Ungeziefermittel gute Dienste ${ }^{10}$ ).

Cit o ba r y u m (Merck) ist ein ,an spezifisch leichte Körper angekittetes " und infolgedessen in Mischung mit schleimigen Vehikeln nicht so leicht sich absetzendes reines Bariumsulfat für die Röntgentechnik ${ }^{11}$ ).

Als neues polytropes Jodpräparat verdient das Ali val (Höchst) Beachtung. Es ist Joddihydroxypropan,

$$
\mathrm{CH}_{2} \mathrm{~J} \cdot \mathrm{CH} \cdot \mathrm{OH} \cdot \mathrm{CH}_{2} \mathrm{OH} \text {, }
$$

steht also chemisch dem Jothion der Elberfelder Farbenfabriken - Dijodhydroxypropan - nahe, ist aber nicht wie dieses eine Flüssigkeit, sondern stellt bei $48-49^{\circ}$ schmelzende, in Wasser wie in fetten Ölen leicht lösliche Krystalle dar. Alival hat vor dem Jothion den Vorzug, daß̉ es sich leicht und schmerzlos injizieren läßt; im übrigen kann die Anwendung auch innerlich, ebenso als Einreibung erfolgen. Es wird dem Alival nachgerühmt, daß es nicht so rasch ausgeschieden werde wie die Jodalkalien, und daß auch seine Verteilung in den einzelnen Organen eine vollkommenere sei; Nebenwirkungen sollen ihm völlig fehlen. Dosis $0,3 \mathrm{~g}$ mehrmals täglich oder $1 \mathrm{~g}$ intramuskulär ${ }^{12}$ ).

An neu auf den Markt gebrachten Gichtmitteln sei zunächst das Artamin erwähnt, Phenylcinchoninsäure „Neumann" (Dr. Neumann \& Co., G. m. b. H., Charlottenburg), mit Atophan chemisch identisch, aber nach anderem Verfahren hergestellt; die Auslegung der Patentanmeldung ist zurzeit noch nicht erfolgt. Unter dem Namen G e l o p o l (Pohl, Danzig) kommt das Präparat in Geloduratkapseln in den Handel ${ }^{13}$ ).

Im Hexophan (Höchst), der Oxykarboxyphenylchinolinsäure,

$$
\overbrace{\mathrm{N}}^{\mathrm{COOH}} \mathrm{C}_{6} \mathrm{H}_{3}<\underset{\mathrm{COOH}}{\mathrm{OH}}+\mathrm{H}_{2} \mathrm{O}
$$

soll die Wirkung des Atophans durch den diuretischen Effekt der Salicylsäurekomponente unterstützt werden. Das Präparat wird durch Kondensation von Acetosalicylsäure mit Isatin gewonnen und bildet. ein gelbes Pulver ohne Geruch und Geschmack. Es wird in Gaben von $1 \mathrm{~g}$ 3-4 mal täglich bei Gicht und Rheumatismus empfohlen, in Form des leichter löslichen Natriumsalzes, $\mathrm{C}_{17} \mathrm{H}_{9} \mathrm{NO}_{5} \mathrm{Na}_{2}+10 \mathrm{H}_{2} \mathrm{O}$, auch zu Injektionen $(0,5 \mathrm{~g}$ pro dosi).

Aguttan (Athenstädt \& Redeker, Hemelingen) ist der von Wolffens tein nach D. R. P. $281007^{13}$ ) hergestellte Orthooxychinolinsalicylsäureester. Für seine Einführung war der Gedanke maßgebend, daß die spezifische Wirkung des Atophans im wesentlichen eine Funktion der Chinolinkomponente sei, und daß das o-Oxychinolin, als einfachster Vertreter der Chinolinreihe, diese Wirkung ebenfalls haben müßte. Wie Brugs oh und W ol f f e n s t e in feststellten, besitzt das o-Oxychinolin tatsächlich die beiden Wirkungen des Atophans, sowohl die „mobilisierende", d. i. die Vermehrung der Ausscheidung fertig gebildeter Harnsäure, wie die ,hemmende" auf die Bildung der Harnsäure; es erwies sich aber gleichzeitig als zu giftig für therapeutische Verwendung. Diese Giftigkeit fehlt dem Salicylsäureester des Oxychinolins, von dem Tagesdosen von 3-4 g und mehr, auf Einzeldosen von $0,5-1 \mathrm{~g}$ verteilt, ohne Nebenwirkungen vertragen wurden; die Wirkung dieses Präparates erstreckt sich nach den genannten Verfassern nur nach einer Richtung, auf die Hemmung der Harnsäurebildung; sie betrachten dies als einen Vorteil, da die beiden konkurrierenden Reaktionen auf den Purinstoffwechsel zu Komplikationen im therapeutischen Erfolg führen könnten ${ }^{14}$ ).

10) Dermatol. Wochenschr. 1915, $337 \mathrm{ff}$.

11) D. Med. Wochenschr. 41, 768 [1915].

$12)$ D. Med. Wochenschr. 41, 648 [1915]; vgl. Angew. Chem.

28. II, 486 [1915].

13) Med. Klinik 11, 1211 [1915].

13a) Angew. Chem. 28, II, 18 [1915].

14) Berl. Klin. Wochenschr. 52, 157 [1915]; Therap. d. Gegenw. 56, 241 ff. [1915]. 
Ebenfalls von Wolffenstein stammt das nach D. R. P. $289001^{15}$ ) hergestellte T or a m in (Athenstädt \& Redeker, Hemelingen), das Ammoniumsalz des Malonsäuretrichlorbutylesters

$$
\mathrm{H}_{2} \mathrm{C}_{\mathrm{COO} \cdot \mathrm{COO} \cdot \mathrm{CH}_{\mathrm{CH}_{3}}^{-\mathrm{CCl}_{8}}}^{\mathrm{CH}_{3}}
$$

Toramin soll in Dosen von $0,1 \mathrm{~g} 5-6$ mal täglich hustenstillend wirken, ohne die Nebenwirkungen der Opiate zu besitzen ${ }^{15 a}$ ).

B a l d r i a n o l (Dr. A. Voswinkel, Berlin) ist Isovalerylcarbamid, also ein entbromtes Bromural, weiße Krystalle vom Fp. $193^{\circ}$, ohne Geruch und Geschmack. Es soll in Dosen von $0,5 \mathrm{~g}$ als Sedativum wirken. Der Körper ist in der Literatur nicht neu, und $\mathrm{S}$ a a $\mathrm{m}$ hatte bei der Einführung des Bromurals bereits darauf hingewiesen, daß die halogenhaltigen Valeriansäurepräparate etwa doppelt so stark wirkten wie die halogenfreien.

Auf dem Gebiet der Opiumalkaloide und Opiumpräparate sind verschiedene Neuerscheinungen zu verzeichnen.

Sowohl Dihydromorphin wie Diacetyldihydromorphin zeigten bei der klinischen Prüfung insofern Vorzüge vor den nichthydrierten Verbindungen, als eine Gewöhnung bei ihnen weniger leicht erfolgte. Das Dihydromorphin ließ sich wegen seiner Reizwirkungen auf den Magen nur als Injektion anwenden, das Diacetyldihydromorphin aber wurde auch innerlich reizlos vertragen und gelangt als $\mathrm{P}$ a $\mathrm{r}$ a $1 \mathrm{a} \mathrm{u} \mathrm{d} \mathrm{i} \mathrm{n}(\mathrm{Knoll})$ in den Handel. Dosis $0,01-0,02$ pro dosi, also mehr wie Heroin ! ${ }^{16}$ )

Die Einführung einer Reihe Konkurrenzpräparate des Pantopons ist nicht ohne politischen Beigeschmack, insofern, als die dieses Präparat darstellende schweizerische Firma in den Geruch deutschfeindlicher Gesinnung geriet und demzufolge mancherlei Angriffe über sich und über ihre Präparate ergehen lassen mußte ${ }^{17}$ ).

So ersetzten beispielsweise die Württembergischen Krankenkassen das Pantopon durch das bereits 1914 eingeführte $\mathrm{L}$ a u d o pan $\mathrm{Ha}$ a $\mathrm{s}$, das laut Deklaration Codein, Morphin, Narcein, Narcotin, Papaverin und Thebain als wasserlösliche mekonsaure Salze enthält; es sind also nicht alle Opiumalkaloide im Laudopan vorhanden. Da B Laudopan „die Lösung des Opiumproblems" bedeute, ist freilich eine recht kühne Behauptung des Prospekts; das Mittel hat nicht einmal Anspruch auf Originalität, da es lediglich die Ideen des Pantopons, des Narcophins und des Laudanons zu kombinieren sucht ${ }^{18}$ )

G l y c o p o n (Chem. Fabrik Nassovia, Wiesbaden) soll die Gesamtalkaloide des Morphins als Glycerophosphate enthalten - die Darstellung ist laut Prospekt zum Patent angemeldet, klinische Mitteilungen aber fehlen über dieses Mittel bisher ebenso wie über I l s o p o n (Dr. Voswinkel, Berlin), das ,die Gesamtalkaloide des Opiums an Salzsäure gebunden" enthalten soll, also mit Pantopon identisch wäre.

Im Gegensatz zu diesen sich an das Pantopon mehr oder weniger eng anlehnenden Präparaten stellt Hol o po n (Chem. Werke vorm. Dr. H. Byk, Oranienburg) etwas wirklich Neues dar. Holopon ist der erste Vertreter der Ultrafiltrate in der Medizin und zugleich das erste ohne weiteres zu Einspritzungen verwendbare galenische Opiumvollpräparat. Mittels Ultrafiltration - Filtration durch eine dünne Kollodiummembran unter Druck - ist es möglich gewesen, einen wässerigen Opiumauszug von den Ballaststoffen der Droge (Schleim, Harz, Fett, Eiweißstoffen usw.) zu befreien, wie sie sowohl in der alkoholhaltigen Opiumtinktur wie in dem trübe löslichen Opiumextrakt in reichlicher Menge vorhanden sind. Im Gegensatz zu diesen beiden Präparaten läßt sich

15) Angew. Chem, 29, II, 7 [1916]

15 a) Berl. Klin. Wochenschr. 5\%, 873 [1915].

16) D. Med. Wochenschr. 41, 846 [1915]

17) Münchn. Med. Wochenschr. 62, 1708 [1915]

18) Müinchn. Med. Wochenschr. 61, 2129 [1914].
Holopon, eine klare hellbraune Flüssigkeit, ohne weiteres injizieren; es entspricht in der Stärke der Opiumtinktur, 10 Teile sind also $=1$ Teil Opium. Im Handel befindet es sich außer als Flüssigkeit auch verarbeitet zu Tabletten und Suppositorien. Die Wirkung ist eine rasch eintretende und lange anhaltende volle Opiumwirkung. Die Dosierung ergibt sich aus dem Opiumgehalt ${ }^{19}$ ).

Im Polyg a l y s t (Bürger-Wernigerode) ist die Senegawurzel erstmalig in Form eines Dialysats gebracht worden; bisher war man auf Dekokt und Extrakt angewiesen. Polygalysat soll, 10-12 tropfenweise genommen, als Expektorans dienen ${ }^{20}$ ).

Das schwedische Dig i t ot a l - nebenbei gesagt das einzige neue ausländische Präparat, über das im Berichtsjahr ein Originalartikel in der deutschen medizinischen Fachpresse erschien - soll alle Glykoside der Digitalis enthalten, aber kein Digitonin. Nähere Angaben über die Gewinnung fehlen. Aus den vorliegenden Mitteilungen läßt sich schlieBen, daß es sich hier um ein Präparat von der Art des Digalens bzw. des Digifolins handelt ${ }^{21}$ ).

Th e a c yl on (Merck), das nach D. R. P. 252641 hergestellte Acetylsalizoyltheobromin

$$
\begin{gathered}
\mathrm{OC} \cdot \mathrm{C}_{6} \mathrm{H}_{4} \cdot \mathrm{OCOCH}_{3} \\
\mathrm{~N}-\mathrm{CO} \\
\stackrel{\mathrm{CO}}{\mathrm{C}}-\mathrm{N} \\
\stackrel{\mathrm{CH}}{3} \\
\mathrm{CH}_{3} \mathrm{~N}-\mathrm{C}-\mathrm{N}
\end{gathered}
$$

wird als dem Theocin in der Wirkung gleichstehendes Diureticum empfohlen. Im Gegensatz zu den bekannten Doppelverbindungen des Theobromins mit Natriumsalicylat bzw. -acetat besitzt das Mittel keinen unangenehmen Geschmack und erzeugt auch keine Ätzwirkung. Im Magen unlöslich, wird es erst im Darm in seine Komponenten gespalten. Dosis : $3-8$ mal täglich $0,5 \mathrm{~g}^{22}$ ).

Das Hexal, primäres sulfosalicylsaures Hexamethylentetramin, sollte die bekannte harndesinfizierende Wirkung des Hexamethylentetramins mit der adstringierenden und sedativen der Sulfosalicylsäure verbinden. Es zeigte sich aber, da $B$ in medizinalen Dosen die erstgenannte Wirkung zu wenig zur Geltung kam. Deshalb wird neuerdings als $\mathrm{N}$ e o h e x a 1 (Riedel) das sekundäre Salz in den Handel gebracht, in dem $2 \mathrm{Mol}$. Hexamethylentetramin an $1 \mathrm{Mol}$. Sulfosalicylsäure gebunden sind. Dosis: $3-6$ mal täglich l g. Dem Mittel wird gegenüber dem Hexal auch bessere Bekömmlichkeit nachgerühmt.

T e s t o g a n (Dr. G. Henning, Berlin) ist nach Angaben des Darstellers ein von Lipoiden und Eiweiß befreiter steriler Auszug aus Stierhoden (400\%ig), T h e l y g a $n$ ein ebensolcher aus Kuhovarien; beiden ist je $1 / 2 \%$ Yohimbin zugesetzt. Die Anwendung dieser Präparate gegen sexuelle Insuffizienz soll nach $\mathrm{I}$ w a $\mathrm{B} / \mathrm{och}$ zwar längere Zeit in Anspruch nehmen, aber meist einen nachhaltigen Erfolg verbürgen. Zu einer Kur sind erforderlich 40 Injektionen von je 2,1 com oder 60-70 Tabletten, entsprechend je $4 \mathrm{~g}$ frischer Drüse und $6 \mathrm{mg}$ Yohimbin ${ }^{23}$ ).

Nur dem Namen nach sei hier G y nor mon (Dr. $H$. Wolff, G. m. b. H., Elberfeld) erwähnt, ein Ovarialpräparat, wie schon viele andere im Handel sind; $\mathrm{M}$ e $\mathrm{n}$ o g e $\mathrm{n}$ heißt dasselbe Mittel in Kombination mit Arsenmetaferrin. Beide Präparate sind zur innerlichen Darreichung bestimmt.

Das Bestreben, das Salvarsan immer weiter auszubauen, führte zum Salvarsan-Natriu m (Höchst). Seine Einführung ist darauf zurückzuführen, daß verschiedene Kliniker sich mit dem neutral reagierenden Neosalvarsan nicht befreunden konnten, sondern das Altsalvarsan in einer beim einfachen Auflösen sofort gebrauchsfertigen Form haben sollten. Salvarsan-Natrium (Präparat 1206) ist das

19) Berl. klin. Wochenschr. 53, 36 [1916].

.0) D. Med. Wochenschr. 41. 1523 [1915].

21) Therap. Monatsh. 89, 611 [1915].

22) Münchn. Med. Wochenschr. 62, 1108 [1915].

23) Med. Klinik 11, 215 [1915]. 
Dinatriumsalz des Altsalvarsans. Man gewinnt es durch Umsetzen dieses letzteren mit Natriumbydroxyd und isoliert es aus der Lösung durch geeignete Fällungsmittel. Es ist wie Salvarsan ein goldgelbes Pulver, gegen Luftsauerstoff ebenso empfindlich wie jenes. Zur Anwendung wird es in $0,4 \%$ iger Kochsalzlösung gelöst. Im übrigen gelten für Salvarsan-Natrium alle die Vorsichtsmaßregeln, die bei Salvarsan selbst zu beachten sind. Es ist hauptsächlich zur intravenösen Injektion bestimmt ${ }^{24}$ ).

An neuen Darmadstringenzien ist zunächst zu nennen E t e l e n (Bayer), Trigallacetol bzw. Gallussäuretriacetyläthylester [D. R. P. $279958^{25}$ )], ein weißes, geschmackfreies Pulver, das vor dem Tannigen, Diacetyltannin, nicht nur den Vorzug der Tropenfestigkeit besitzen, es vielmehr auch in der Stärke der Wirksamkeit übertreffen soll ${ }^{25 a}$ ).

Ferner Noventerol (Dr. W. Wolff, G. m. b. H., Elberfeld), das Aluminiumsalz einer Tannineiweißverbindung. Das Deutsche Arzneibuch verlangt von seinem Tanninalbuminat (Tannalbin), daß bei der künstlichen Magenverdauung nicht über $50 \%$ gelöst würden. Noventerol wird dem gegenüber nach $\mathrm{B}$ a $\mathrm{c} \mathrm{h}$ e $\mathrm{m}$ vom Magensaft nur $\mathrm{zu}$ $20 \%$ angegriffen; im Darmsaft löst es sich völlig auf; die Aluminiumkomponente soll die adstringierende Wirkung des Tanninalbuminats noch unterstützen ${ }^{26}$ ).

Die Behandlung von Darmaffektionen mit adsorbierenden Substanzen erfreut sich steigender Beliebtheit. Bolus, frisch gefälltes Aluminiumhydroxyd, Tierkohle, amorphe Kieselsäure sind für sich oder in den verschiedensten Kombinationen miteinander empfohlen und als Spezialitäten in den Handel gebracht worden.

Bei der Tierkohle muß freilich besonders darauf geachtet werden, daß genügend gereinigte Präparate zur Anwendung kommen, da nur solche genügende Adsorptionskraft besitzen. Solche besonders gereinigte Präparate sind z. B. im Berichtsjahre als Carbo a $\mathrm{nimalis} \mathrm{Merck}$ und $\mathrm{C}$ a r b o v e n t (Österr. Verein. f. chem. u. metallurg. Produkte, Wien) auf den Markt gekommen.

Úber Pankrofirm Dr. S c h e e r messer (Münchener Pharm. Fabrik München), ein ,,an natürliches Milcheiweiß gekuppeltes Pankreaspräparat, frei von Tannin und unerwünschten Nebenwirkungen", fehlen einstweilen noch klinische Mitteilungen. Der Prospekt legt großen Wert auf die Abwesenheit des Tannins, das die Pankreatinwirkung beeinträchtige - das bedeutet also einen indirekten Angrif auf das bekannte tanninhaltige Pankreon der Rhenania.

Die Darreichung löslicher Kalksalze wird neuerdings zur Erzielung eines lebhafteren Stoffwechsels vielfach empfohlen. Diese Calciumtherapie führte zur Schaffung zahlreicher Präparate und Spezialitäten. Es seien hier genannt Glykalzium effervescens Ritsert (Dr. Ritsert, Frankfurt a. M.), ein Brausesalz, in dem eine Chlorcalciumlactoseverbindung enthalten ist ${ }^{27}$ ), und $\mathrm{K}$ a $\mathrm{l} \mathrm{zan} \mathrm{(J.A.}$ Wülfing, Berlin), 0,5 g schwere Tabletten aus CalciumNatriumlactat ${ }^{28}$.

$\mathrm{Zu}$ den bisher genannten Mitteln, die einen mehr oder minder großen Anspruch auf Originalität besitzen, kommen nun noch verschiedene, die als Ersatz von Präparaten und Arzneispezialitäten des feindlichen Auslands bestimmt sind So bringt die Firma Heyden z. B. an Stelle der französischen Tiodine ein $J$ od ät h y l-Thiosi n a m in in den Handel und an Stelle des noch zu erwähnenden Enesols ein Hyrgas o I; auch für Electrargol Clin und die Clinschen Kakodylate wurden verschiedene deutsche chemisch identische Präparate herausgebracht.

Mehr Interesse dürften die Compretten bzw. A m $p h$ i o le $n$ M BK verdienen, sie sind als Ersatz der von Burrough, Welcome \& Co. in London hergestellten bekannten „Tabloids" oder Ampullen gedacht, die sich ihrer praktischen und ansprechenden Form und Aufmachung halber bei uns

24) Münchn. Med. Wochenschr. 62, $177 \mathrm{ff} ., 612,1707$ [1915]; D. Med. Wochenschr. 41, 335, 1301 [1915]; Berl. Klin. Wochenschr. 52, 400 [1915]; Apotheker-Ztg. 30, 103 [1915].

25) Angew. Chem. 2r, II, 689 [1914].

25 a) Münchn. Med: Wochenschr. 62, 1745 [1915].

26) Med. Klinik 11, 808 [1915].

27) Therap. Monatsh. 29, 293 [1915]

28) Arztl. Rundschau 1915, Nr. 35. großer Beliebtheit erfreuten. Die Firmen Merck, Boehringer und Knoll, die diese Zubereitungen gemeinsam in den Handel bringen, baben sich indes nicht auf die gebräuchlichsten entsprechenden Präparate von Burrough, Welcome \& Co. beschränkt, sondern haben fortbauend noch eine ganze Reibe anderer Präparate in jener bequemen Aufmachung berausgebracht. Als neu seien hier z. B. die zum Auflutschen bestimmten Chlorcalci u kompretten genannt, die eine angenehme Darreichung dieses übelschmeckenden Arzneistoffs bei Heufieber ermöglichen ${ }^{29}$ ).

Es mutet demgegenüber eigenartig an, zu sehen, wie sich das feindliche und zum Teil auch das neutrale, von der deutschen Zufuhr abgeschnittene Ausland bemüht, dem Mangel an deutschen Arzneipräparaten abzuhelfen. In der Fachpresse und auch in den Tageszeitungen ist zur Genüge darüber berichtet worden, zu welchen Auswüchsen dieses Bestreben geführt hat, wie z. B. statt Acetylsalicylsäure doppeltkohlensaures Natrium, statt Salvarsan gefärbtes Kochsalz oder bestenfalls gefärbtes kakodylsaures Natrium untergeschoben wurde.

Bedenkt man freilich, daß ein recht hoher Prozentsatz „normaler" ausländischer, namentlich französischer Arzneipräparate und Spezialitäten seit Jahr und Tag falsch deklariert unter bewußt falscher Flagge segelte ${ }^{30}$ ), so erscheinen solche Entgleisungen minder erstaunlich. Erst unlängst wurde z. B. wieder das bei uns früher ziemlich oft angewandte En e s o l (Clin) entlarvt: der angeblicho saure Salicylsäureester der Arsensäure erwies sich als ein Gemisch aus methylarsinsaurem Natrium mit Hydrargyrum salicylicum; der Quecksilbergehalt betrug noch nicht die Hälfte des angegebenen ${ }^{31}$.

Es besteht aller Grund zu der Annahme, daß das $\mathrm{Ab}$ hängigkeitsverhältnis des Auslands vom deutschen Arzneimittelmarkt auch nach dem Friedensschluß weiter bestehen bleiben wird. Selbst gesetzt den mehr als unwahrscheinlichen Fall, daß es dem Ausland gelingen sollte, sich während des Krieges durch Einrichtung eigener Fabrikation in chemisch-pharmazeutischen Präparaten von uns unabhängig zu machen, so bleibt doch kaum ein Zweifel, daß unsere überlegene Technik in kurzer Frist diese ausländischen Produktionsstätten überholen und aufs neue aus dem Felde schlagen. wird.

[A. 8.]

\section{Beiträge zur Kenntnis militärischer Ausrüstungsgegenstände.}

Von Dr. Eugen Seet und Dr. Albert Sander in Stuttgart. (Eingeg. 24./1. 1916.)

\section{Chemische und physikalische Untersuchungen feldgrauer Sommerstoffe ${ }^{1}$ ).}

Im letzten Sommer sind von der Militärverwaltung neben den herkömmlichen feldgrauen Tuchen auch feldgraue Sommerstoffe aus Baumwolle für die Sommeruniformen zugelassen worden.

Später wurden diese baumwollenen Bekleidungsstücke ausschließlich als leichte Arbeitsanzüge an Stelle von Drillich an die Bekleidungsolepots ins Feld ausgegeben. Die Lieferungsbedingungen für diese Stoffe seien zur rascheren Orientierung hier kurz angeführt.

Das Gewicht des laufenden Meters ist für die Breite von $70 \mathrm{~cm}$ auf $325 \mathrm{~g}$ beim Hosenstoff und auf $210 \mathrm{~g}$ beim Rockstoff festgesetzt. Bei doppelter Stoffbreite wird dementsprechend das doppelte Gewicht verlangt.

Bei beiden Stoffarten soll die gleiche Garnnummer (24/2) für die Kette verwendet werden. Für den Sehuß ist beim Hosenstoff die Garnnummer 12, beim Rockstoff die Nummer 10 vorgeschrieben. Beide Stoffarten sollen auf der linken Seite des angenehmeren Tragens wegen leicht angerauht sein, um sie etwas wärmer und

29) Münchn. Med. Wochenschr. 62, 912 [1915]

30) Vgl. D. Med. Wochenschr. 41, 501 [1915].

31) Pharm. Ztg. 60, 724 [1915].

1) I. Chemische und mikroskopische Untersuchungen feldgraver Tuche. Angew. Chem. 28, I, 1457-1463 [1915]. 